\title{
Observations of Tempel's Periodic Comet of six years, made at the Cordoba Observatory.
}

On the 21 st. of June 1879 I received, through the courtesy of Dr. Gautier, his new ephemeris of this comet, and by this means the comet was easily found on the same evening. It was very faint, circular, $1 \frac{1}{2^{\prime}}$ or $2^{\prime}$ in diameter, and with a perceptible condensation toward the center.

Observations were secured by Mr. Thome on six consecutive nights, after which they were impossible with this equatorial of 28 centimeters aperture. The brightness on June 21 was comparable with that of a star of the 13th magnitude. On June 26 it could not be seen with the faintest illumination of the threads until at least half an hour after it was found. Hence it would be unreasonable to expect great precision in the observations. The comparison-stars, two of which occur in Argelander's zones, have all of them been determined here

\begin{tabular}{|c|c|c|c|c|c|c|c|c|}
\hline \multirow{2}{*}{\multicolumn{2}{|c|}{$\begin{array}{l}\text { Date } \\
1879\end{array}$}} & \multirow{2}{*}{$\begin{array}{l}\text { Cordoba } \\
\text { M. T. }\end{array}$} & \multirow{2}{*}{ N.Obs } & \multirow{2}{*}{ Comp.-Star } & \multicolumn{2}{|c|}{$\mathscr{E}-*$} & \multicolumn{2}{|c|}{ Comet's apparent } \\
\hline & & & & & $\Delta a$ & $A \delta$ & $a$ & $\delta$ \\
\hline \multirow[t]{6}{*}{ June } & 21 & $8^{\mathrm{n}} 27^{\mathrm{m}} 35^{\mathrm{s}} 6$ & 10 & OA. 15783 & $-1^{\mathrm{m}} 10^{\mathrm{s}} 0$ & $+1^{\prime} 22^{\prime \prime} 3$ & $16^{\mathrm{h} 29^{\mathrm{m}} 5 \mathrm{gs}^{2}} 5$ & $-22^{0} 37^{\prime} 34$ \\
\hline & 22 & 72613.6 & 9 & (1) & -011.1 & -126.2 & 2940.0 & $22 \quad 4651$ \\
\hline & 23 & $\begin{array}{lll}8 & 19 & 20.8\end{array}$ & 9 & O A. 15781 & -137.8 & +419.1 & 9922.5 & $22 \quad 55 \quad 47$ \\
\hline & 24 & 72956.2 & 10 & $"$ & -152.7 & -517.8 & $29 \quad 7.6$ & $23 \quad 624$ \\
\hline & 25 & $\begin{array}{lll}8 & 15 & 53.4\end{array}$ & 10 & (2) & -217.2 & -845.3 & 2853.2 & 231636 \\
\hline & 26 & 82757.3 & 7 & (3) & -429.1 & $\begin{array}{ll}-1 & 3.4\end{array}$ & $1628 \quad 41.5$ & $-23 \quad 2552$ \\
\hline
\end{tabular}

Observed Mean Places of the Comparison Stars, for Equinox 1879.0

\begin{tabular}{|c|c|c|c|c|}
\hline Name & Mag. & Nr. Obs. & $\epsilon$ & $\delta$ \\
\hline OA. 15783 & $81 / 4$ & 4 & $16^{\mathrm{b}} 31^{\mathrm{m}} \quad 4^{\mathrm{s}} 36$ & $-22038^{\prime} 44^{\prime \prime} 4$ \\
\hline (1) & 10 & 4 & 2946.95 & $\begin{array}{lll}22 & 45 & 12.3\end{array}$ \\
\hline O A. 15781 & $91 / 4$ & 5 & 3056.14 & $23 \quad 0 \quad 542$ \\
\hline$(2)$ & $101 / 2$ & 3 & $\begin{array}{ll}31 & 6.28\end{array}$ & $\begin{array}{lll}23 & 7 & 38.8\end{array}$ \\
\hline (3) & $9^{3} / 4$ & 4 & 1633 & $\begin{array}{llll}-23 & 24 & 36.4\end{array}$ \\
\hline
\end{tabular}

Cordoba 1880, April 5.*)

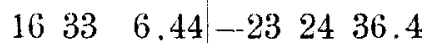

*) Ein von Herrn Dr. Gould am 17. August v. J. an die Redaction gesandtes Schreiben, welches die obigen sowie die in Nr. 2320 abgedruckten Beobachtungen von Vergleichsternen des Encke'schen Cometen enthielt, ist hier nicht angekommen. Anm. der Red.

\section{Literarische Anzeige.}

Publications of the Cincinnati observatory. Micrometrical measurements of double stars. 1878-79.

Von 1054 beobachteten Doppelsternen gehören 622 der südlichen, 432 der nördlichen Halbkugel an. Gegen 200 sind neu. In der Einleitung behandelt Ormond Stone, Director der Sternwarte in Cincinnati, die Beobachtungsfehler. Beobachter waren O. Stone, H. A. Howe und H. V. Egbert.

$L$.

\section{In $h$ a $1 \mathrm{t}$ :}

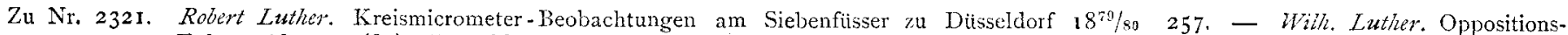
Ephemeride von (84) Klio 1880. 263. - Th. Bredichis. Schreiben an den Herausgeber. 265. - 7. M. Schaeberle. Elemente und Ephemeride des Cometen $b 1880$ (schreiben an den Herausgeber). 265. - Fohn Teboutt. Observations of Pallas at Windsor. N. S. Wales. 269. - Berichtigungen. 27I. - Literarische Anzeigen. 27 I.

Zu Nr. 2322. H. A. Howe. A new approximate Solution of Kepler's Problem. 273. - C. Bruhns. Ableitung der Elemente des Kometen I, 1878. 277. - Pietro Tacchini. Osservazioni di pianeti e ultime osservazioni della cometa 6 1880. 279. - W. Doberck. Elements of $\zeta$ Cancri, 283 . - W. Doberck. Formulae for some double star. 285 . B. A. Goutd. Observations of Tempel's Periodic Comet of six years. 287. - Literarische Anzeige. 287. 